\title{
61. DOS ORQUÍDEAS NUEVAS PARA LA FLORA DE LA COMUNIDAD VALENCIANA
}

\author{
Joan PIERA y Manuel B. CRESPO
}

Two orchids new for the flora of the Valencian Community

Palabras clave. Corología, Orchidaceae, Alicante, Valencia, España.

Key words. Corology, Orchidaceae, Alicante, Valencia, southeastern Spain.

Como consecuencia de nuestras herborizaciones por las comarcas del norte de Alicante y sur de Valencia, hemos descubierto dos interesantes orquídeas novedosas para la flora de la Comunidad Valenciana. A continuación se ofrecen algunos datos de interés sobre ambas, que complementan lo que avanzábamos en reciente publicación (Mateo y Crespo, 1995: 418-425).

\section{Orchis purpurea Hudson}

Hs, *VALENCIA: Bocairent, cerca de Casetes Noves del Pi, en la Sierra de Mariola, 30SYH0991, 850 m, 25-IV-1992, Piera (ABH 4421).

Interesante taxón de amplia distribución eurasiática (Pignatti, 1982: 717) del que no se tenía conocimiento previo para la flora valenciana (cf. Bolòs \& al., 1990; Mateo \& Crespo, 1990), pese a que su presencia era conocida de diversas provincias de los territorios iberolevantinos colindantes (cf. Rivera \& López-Vélez, 1987: 125; Bolòs et al., 1990; Mateo, 1990: 472). Con esta referencia se completa la información que de modo fotográfico se ofrecía en Masalles et al. (1988: 375), correspondiente a individuos de la población indicada.

En la nueva localidad persiste una población de algo más de treinta individuos, cuyo seguimiento se viene realizando ininterrumpidamente desde hace varios años. Orchis purpurea se presenta en los claros de una masa boscosa constituida principalmente por Quercus rotundifolia Lam., Pinus pinea L., Juniperus oxycedrus L., Erinacea anthyllis Link, Salvia blancoana subsp. mariolensis Figuerola, Lavandula latifolia Medik., Ophrys scolopax Cav., etc. Fitosociológicamente, se integra en fragmentos de vegetación de la serie mesomediterránea manchega, murciana, aragonesa, setabense y valencianotarraconense, basófila y seca-subhúmeda de la carrasca (Querceto rotundifoliae Sigmetum) en su subserie setabense (ulicetoso parviflori sigmetosum). Dada la proximidad de esta localidad al límite provincial con Alicante, es muy probable su existencia en esta última provincia.

Himantoglossum hircinum (L.) Spreng. subsp. hircinum

[三Loroglossum hircinum (L.) L.C.M. Richard]

Hs, *ALICANTE: Tàrbena, El Somo, km. 7 de la carretera a Castell de Castells, 30SYH4889, 800 m, 23-VI-1995, Beviá, Ferrer, Yébenes \& Piera (ABH 13610).

Taxón de distribución eminentemente Mediterráneo centro-occidental y Atlántica (Moore, 1980: 342) que se presenta disperso por buena parte de la mitad occidental y norte de la Península Ibérica (Fernández Casas et al., 1992: 174); pero que hasta el momento no 
se conocía en la flora de la Comunidad Valenciana (Bolòs et al., 1990; Mateo y Crespo, 1990).

En la nueva localidad se presenta una población con cerca de un centenar de individuos que florecen y fructifican con normalidad. En ella, H. hircinum aparece junto a Brachypodium retusum (Pers.) Beauv., Teucrium chamaedrys L., Carduncellus monspclionsium All., Asphodelus ramosus L., Thapsia villosa L., Aphyllanthes monspeliensis L., Leucanthemum gracilicaule (Duf.) Pau, Phlomis lychnitis L., Catananche caerulea L., Ononis minutissima L., Chamaerops humilis L., Digitalis obscura L., Rubia peregrina L. subsp. longifolia (Poiret) O. Bolòs, Hedera helix L., Scorzonera ang ustifolia L., Euphorbia isatidifolia Lam., Cistus albidus L., Teucrium flavum L. subsp. glaucum (Jordan \& Fourr.) Ronniger, Teucrium homotrichum (Font Quer) Rivas Mart., etc.; con los que participa en comunidades de transición entre lastonares de Teucrio pseudochaemaepitys-Brachypodietum retusi O. Bolòs 1957 (Cl. Lygeo-Stipetea Rivas Mart. 1978) y fenalares de Lathyro tremolsianiBrachypodietum phoenicoidis Costa \& al. 1985 (Cl. Festuco-Brometea Br.-Bl. \& R. Tüxen 1943). Dicha formación vegetal constituye una etapa inicial de regeneración tras los incendios ocurridos entre 1990 y 1991, que corresponde a la serie termomediterránea valencianotarraconense, setabense y murcianoalmeriense, basófila y seco-subhúmeda de la carrasca (Rubio longifoliae-Querceto rotundifoliae Sigmetum).

\section{BIBLIOGRAFÍA}

BOLÒS, O., J. VIGO, R. M. MASALLES y J. M. NINOT -1990- Flora Manual dels Països Catalans. Pòrtic. Barcelona.

FERNÁNDEZ CASAS, J., R. GAMARRA y M.J. MORALES -1992- De flora iberica index chartographicus. Treb. Inst. Bot. Barcelona 15: $1-422$.
MASALLES, R.M., J. CARRERAS, A. FARRÀS, J.M. NINOT y J.M. CAMARASA -1988Història Natural dels Països Catalans. 6. Plantes superiors. Enciclopèdia Catalana. Barcelona.

MATEO, G. y M.B. CRESPO-1990- Claves para la flora valenciana. Del Cenia al Segura. Valencia.

MATEO, G. y M.B. CRESPO -1995-Flora Abreviada de la Comunidad Valenciana. Gamma. Alicante.

MATEO, G. -1990- Catálogo florístico de la provincia Teruel. Instituto de Estudios Turolenses. Teruel.

MOORE, D.M. -1980- Himantoglossum Koch. In: Tutin, T. G. \& al. (eds.), Flora europaea 5: 342. Cambridge University Press. Cambridge.

PIGNATTI, S. -1982- Flora d'Italia 3. Edagricole. Bolonia.

RIVERA, D. y G. LÓPEZ-VÉLEZ - 1987- Orquideas de la provincia de Albacete. Albacete.

Aceptado para su publicación en Marzo de 1997

Dirección de los autores. J.PIERA: Seminario Didáctico de Ciencias Naturales. Instituto de Bachillerato Mix to de Altea. Partida Garganes, s/n. E-03590 Altea (Alicante). M.B.CRESPO: Departamento de Ciencias Ambientales y Recursos Naturales (Botánica). Universidad de Alicante. Apartado 99. E-03080 Alicante. 